\title{
Control Strategies of Contagion Processes in Time-varying Networks
}

\author{
Márton Karsai and Nicola Perra
}

\begin{abstract}
The vast majority of strategies aimed at controlling contagion processes on networks consider a timescale separation between the evolution of the system and the unfolding of the process. However, in the real world, many networks are highly dynamical and evolve, in time, concurrently to the contagion phenomena. Here, we review the most commonly used immunization strategies on networks. In the first part of the chapter, we focus on controlling strategies in the limit of timescale separation. In the second part instead, we introduce results and methods that relax this approximation. In doing so, we summarize the main findings considering both numerical and analytically approaches in real as well as synthetic time-varying networks.
\end{abstract}

\section{Introduction}

A wide range of real-world phenomena such as the spreading of ideas, memes, infectious diseases, and malwares can be effectively modeled as contagion processes on networks $[1,2,3,4,5]$. An intense research on the subject allowed to identify a set of network features that affect such processes. In particular, two properties have been thoroughly investigated. The first is the heterogeneity observed in the distribution of networks' metrics. Examples are the number of connections per node, degree, and the intensity of contacts, weight. These quantities follow distributions characterized by heavy-tails, which imply the absence of characteristic scales and the presence of large fluctuations respect to the average [6]. The second is the higher-order organi-

Márton Karsai

Univ de Lyon, ENS de Lyon, INRIA, CNRS, UMR 5668, IXXI, 69364 Lyon, France, e-mail: marton.karsai@ens-lyon.fr

Nicola Perra

Centre for Business Network Analysis, University of Greenwich, Park Row, London SE10 9LS, United Kingdom, e-mail: n.perra@greenwich.ac.uk 
zation of connectivity patterns associated to the presence of clusters/communities and or to the correlation between node's features $[2,7]$.

The understanding of these properties and their effects on spreading phenomena has spurred the creation of strategies aimed at controlling or promoting diffusion processes. These can be classified in two main categories [8]. In the first, we find global strategies that rely on the full knowledge of the network structure. In the second instead, we find local strategies which relax this, often unrealistic, assumption. In order to better understand the problem set-up, let's imagine we want to protect a network of computers against the spreading of malwares. The trivial solution is clearly to immunize all computers. However, this strategy is very costly and often unpractical. The problem then is finding a way to immunize just a fraction $p$ of nodes and still effectively protect the entire network. Each prescription for the selection of this fraction constitutes what we call a strategy. To this end, global strategies use centrality measures such as degree, $\mathrm{k}$-core, betweenness and pagerank to rank the importance of each node $[1,2,9,10]$. Local strategies instead infer the role of nodes by local explorations and samples [11].

One common assumption in the majority of related works is to consider a timescale separation between the changes in network structures, $\tau_{G}$, and the contagion process $\tau_{P}$. Indeed, spreading processes have been typically considered to take place in either static $\left(\tau_{P} \ll \tau_{G}\right)$ or annealed $\left(\tau_{P} \gg \tau_{G}\right)$ networks. While this approximation can be used to study a range of processes such as the spreading of some diseases in contact networks or the propagation of energy in power grids it fails to describe many others phenomena in which the two timescales are comparable $[12,13,14,15,16,17,18,19,20,21,22,23,24,25,26,27,28,29,30,31,32$, $33,34,35,36,37,38,39,40]$. In these cases, such as the spreading of ideas, memes, information and some type of diseases the diffusion processes take place in timevarying networks $[41,42,43]$. Thus their study and modeling needs to consider the interplay between the two simultaneously unfolding dynamics.

In this chapter, we will first review the main strategies to control contagion processes in static and annealed networks. We will then focus on time-varying graphs and discuss the recent body of research based on numerical simulations of contagious processes on real and synthetic temporal networks. Finally, we will consider spreading processes unfolding on a particular class of time-varying networks called activity-driven networks which allows the analytical study of different global and local strategies of immunization. As prototypical example of contagion processes we will consider the Susceptible-Infected-Susceptible (SIS) model [44, 45, 1]. SIS dynamics are characterized by two transitions between the two different mutually exclusive states of nodes $S+I \stackrel{\beta}{\rightarrow} 2 I, I \stackrel{\mu}{\rightarrow} S$. The stationary state of the process is described by two different behaviors. Above a threshold an endemic state is reached where the density of nodes in the class $I, I_{\infty}$, is fixed by the balance of the spreading and recovery rates. Below the threshold the spreading is not able to sustain itself and dies out, thus in the stationary state of the process the number of nodes in the class $I$ is zero. 


\section{Controlling contagion processes in static/annealed networks}

How can we protect a network from the spreading of an infectious disease? While this is the underlying question of the chapter, in this section we will first focus on two timescale separation limits. In order to devise effective control strategies it is crucial to understand how contagion processes spread on networks. In particular, it is important to determine how the properties of networks affect such phenomena. As mentioned above, the topology of real-world networks is typically characterized by broad degree distributions often approximated by power-laws i.e. $P(k) \sim k^{-\gamma}$. The heterogeneities that these distributions entail have genuine effects on the unfolding of contagion processes. Indeed, it has been shown [46] that the epidemic threshold of a SIS process spreading on uncorrelated annealed networks is:

$$
\frac{\beta}{\mu}>\frac{\langle k\rangle^{2}}{\left\langle k^{2}\right\rangle}
$$

The derivation of the threshold is performed by means of heterogeneous mean-field (HMF) theory which assumes that nodes with the same degree are statistically equivalent. In this perspective connections between nodes are continuously reshuffled such that the degree distribution $P(k)$ and the probability of having connections between nodes with degree $k$ and $k^{\prime}, P\left(k^{\prime} \mid k\right)$, are kept constant [15]. The timescale describing this shuffling is much faster than the timescale describing the spreading process. Consequently, the disease spreads in an effective network where all these connections are available routes of contagion. Interestingly, in the case of networks with exponent $2<\gamma<3$ the second moment of the degree distribution diverges as $N \rightarrow \infty$, which implies a vanishing epidemic threshold: any virus with a spreading rate larger than zero will be able to affect a macroscopic fraction of the network. In finite networks where only finite fluctuations are possible, none of the moments diverge thus the threshold is larger than zero. However, the presence of nodes with degree much larger than the average, hubs, pushes the threshold to values much smaller than in the case of corresponding homogeneous networks with the same size (number of nodes) and average connectivity. Indeed, hubs decrease the shortest paths between any pair of nodes in the network and once infected, they dramatically accelerate the diffusion by virtue of their large number of connections.

In case of static networks the timescale describing the evolution of the contagion process is much faster than the timescale of the network, thus the contagion phenomena evolves in a frozen, quenched, structure. The threshold of an SIS model in this case is tied to the spectral properties of the adjacency matrix $A_{i j}$ coding for the static network structure. Indeed, for an arbitrary network the epidemic threshold is inversely proportional to the largest eigenvalue $\lambda_{1}[47,48,49]$ of the matrix. In case of uncorrelated networks, $\lambda_{1} \sim \sqrt{k_{\max }}$, thus the threshold is associated to the largest degree in the system. It is interesting to notice that the analytical results for uncorrelated annealed and static scale-free graphs are equivalent if the degree exponent $\gamma<2.5$, while if $\gamma>2.5$, the results obtained with the HMF theory deviate from those obtained in static networks. Although the study of such deviation is fas- 
cinating, it is beyond the scope of this chapter, we refer the interested reader to the following recent papers on the subject [50, 51, 52, 53].

Now that we understand in some details how contagion phenomena spread on networks we can shift the attention towards controlling/vaccination strategies. Interestingly, this problem is closely related to the robustness of a network structure against attacks [54], which further maps to a site percolation in networks [3]. In both cases the objective is to identify a critical fraction of nodes which removal would segregate the network structure and thus disrupt the spreading of a contagion process. In case of vaccination this translates to the identification of a critical $p_{c}$ fraction of nodes to immunize in order to push the epidemics to an inactive phase i.e. below the threshold. Studies discussed above suggests that nodes in heterogeneous networks have very different roles in sustaining spreading processes and have indisputable effects on the epidemic threshold. In order to demonstrate this observation, let us consider the simplest vaccination strategy which involves a fraction $p$ of randomly selected nodes. This strategy is agnostic to the features of the networks and has been shown to simply re-scale the effective spreading rate $\beta \rightarrow \beta(1-p)$ $[10,55]$. For uncorrelated annealed networks, the threshold becomes:

$$
\frac{\beta}{\mu}\left(1-p_{c}\right)=\frac{\langle k\rangle^{2}}{\left\langle k^{2}\right\rangle} .
$$

In this case, the large fluctuations typical of heavy-tailed degree distributions push the critical immunization fraction $p_{c}$ to values close to one. In other words, in order to protect heterogeneous networks against infectious diseases a random immunization strategy is inefficient as it requires a large fraction of nodes to be vaccinated.

Can we do better, and if yes, how? The answer to this question depends on the amount of information available about the network structure. Let's first consider a scenario in which we have full access to the structure and features of the nodes. In this case, we can implement targeted immunization strategies based on global knowledge. These relies on different centrality measures which have been developed to characterize and rank the importance of nodes in a network [1,2]. Interestingly, many of these are defined via diffusion processes [56], thus the importance of nodes is explicitly associated to their role in sustaining spreading phenomena.

One of the simplest centrality measure is the degree centrality, which indicates hubs in the network. By removing nodes with the highest degree it is possible to reduce degree fluctuations which in turn increases the epidemic threshold. Here the critical fraction $p_{c}$ to arrest the epidemic spreading is determined by a critical $k_{c}(p)$ degree which corresponds (for annealed uncorrelated networks) to the critical point $\langle k\rangle_{p_{c}}^{2} /\left\langle k^{2}\right\rangle_{p_{c}}=\beta / \mu$. For graphs with degree exponent $\gamma=3$ the critical fraction of immunization to arrest epidemics appears as

$$
p_{c} \sim e^{-2 \mu / k_{\min } \beta}
$$

where $k_{\min }$ assigns the smallest degree in the network [10, 55]. As clear from the exponential dependence on the $k_{\min }$ and $\beta$, targeted vaccination strategy based on degree centrality provides a very efficient way to control epidemic spreading. Similar 
conclusions can be drawn by applying other centrality measures, such as betweenness and k-core centrality, or memberships, for the selection of immunized nodes $[9,57,58,59,60,61]$.

Let's consider now the case in which we don't have access to the global structure of the network and to the features of all nodes. Immunization strategies developed under this very realistic assumption are typically called local strategies as they are based on local exploration and sampling of the network structure.

One of the most notable local methods is the acquaintance vaccination [11]. This relies on the so called "friendship paradox": your friends have more friends that you do. In the case of uncorrelated heterogeneous networks is very easy to prove that this counterintuitive fact holds true [1,2]. Several studies performed in real-world networks confirm the paradox as a genuine feature tied to the large fluctuations in degree observed across a wide range of graphs $[62,63,64]$.

Cohen et al realized how this feature can be used to find highly connected nodes in the system via a local exploration. In the acquaintance strategy we first select randomly an $f$ fraction of probe nodes. However, instead of vaccinating them, we choose randomly one of their neighbors as target of our vaccination. Note that the local search from different probes can lead to the same neighbor i.e. $p \leq f$. The critical fraction of nodes is:

$$
p_{c}=1-\sum_{k} P(k) v_{f_{c}}^{k}
$$

where $v_{f}=\left\langle e^{-f / k}\right\rangle_{k}$ is the probability that an acquaintance is not selected in a single attempt, while $f_{c}$ assigns the critical fraction of random probes which can be determined numerically as shown in $[3,11,65,66]$. The critical immunization fraction of this method is always $p_{c}<1$ and considerably smaller for scale-free networks with any degree exponent as compared to the random vaccination strategy [11].

Note that other variations of the acquaintance vaccination strategy were proposed by optimizing the neighbor selection [67]. At the same time immunization was also studied on meta-population networks [68]. In other studies, it has been argued that efficient immunization strategies can be developed by considering the higher-order organization of connectivity patterns $[57,69,70,71,72,73,74]$. Further studies proposed vaccination strategies using evolutionary games [75], or considering complex contagion processes [76]. For a recent review on the subject we refer the readers to Ref. [8].

\section{Controlling contagion processes in time-varying networks}

We now move away from the limits of timescale separation. In fact, we are ready to study spreading processes and immunization strategies on time-varying networks in which the timescales driving the evolution of the system and of the spreading process are comparable. As done before for annealed and static networks, we will first 
study the epidemic threshold. We will then use this knowledge to present and better understand immunization strategies aimed at controlling contagion phenomena.

A closed formula for the epidemic threshold of a SIS epidemic process unfolding on any time-varying has been derived [77]. In this approach the time-varying network is considered as a sequence of adjacency matrices $A_{1}, A_{2}, \ldots, A_{T}$ which describe the evolution of the network in time. Prakash et al showed that the disease will not be able to spread in the system if

$$
\lambda_{\prod_{i} \mathbf{S}_{\mathbf{i}}}<1
$$

where $\mathbf{S}_{\mathbf{i}}=(1-\mu) \mathbf{I}+\alpha \mathbf{A}_{\mathbf{i}}$, and $\alpha$ is the transmission rate per contact. In other words, the disease will die out if the largest eigenvalue of the system-matrix $\mathbf{S}=\prod_{i} \mathbf{S}_{\mathbf{i}}$ is smaller than one. This result have been recently confirmed with a different approach [78]. It is interesting to note that in both static and time-varying networks the largest eigenvalue, of the adjacency matrix in one case and of the system matrix in the other, determines the unfolding of the disease. Despite the generality and the practical importance of this result, the computation of the threshold needs to be done numerically. Also, the condition obtained hinders the effects of temporal connectivity patterns on spreading processes. For this reason, in the next subsections we will consider SIS processes unfolding on a particular type of timevarying networks. In doing so, we will be able to derive a more explicit condition for the spreading and thus better understand the efficiency of different immunization strategies.

\subsection{Epidemic threshold on activity-driven networks}

In activity-driven networks, each node $i$ is characterized by an activity rate $a_{i}$. This quantity encodes the probability per unit time to establish contacts with other nodes. The activity rates are assigned according to a given probability distribution $F(a)$. Observations in a wide range of different real-world networks show that activities follow heavy-tailed distributions $[32,33,29,79,80]$. We approximate such distributions as power-laws, i.e., $F(a)=B a^{-\gamma}$ with activities restricted in the region $a \in[\varepsilon, 1]$ to avoid divergences for $a \rightarrow 0$. In these settings, the generative network evolution process is defined according to the following rules:

- At each discrete time step $t$, the network $G_{t}$ starts with $N$ disconnected vertices;

- With probability $a_{i} \Delta t$, each vertex $i$ becomes active and generates $m$ links that are connected to $m$ other randomly selected vertices;

- At the next time step $t+\Delta t$, all the edges in the network $G_{t}$ are deleted.

All interactions have a constant duration $\Delta t$. In the following, without loss of generality, we will set $\Delta t=1$. Activity-driven networks in their simplest form are random and memoryless. We refer the reader interested in more realistic extensions of the model to the following references $[29,79,80,81]$. The full dynamics of the network and its ensuing structure is completely encoded in the activity distribution $F(a)$. 
Moreover, it is possible to prove that integrating activity driven networks in finite time windows such that $T \ll N$ and $k \ll N$ yield graphs characterized by degree distributions following the functional form $F(a)[32,82]$.

Let's now consider a SIS model unfolding on activity-varying networks. Using the HMF theory we group nodes according to their activity assuming that nodes in the same class are statistically equivalent. At the mean-field level, the spreading process can be described by the number of infected individuals in the class of activity $a$ at time $t$, i.e., $I_{a}^{t}$ [32]. Following Ref. [32], the number of infected individuals of class $a$ at time $t+1$ given by:

$$
I_{a}^{t+1}=I_{a}^{t}-\mu I_{a}^{t}+\alpha m\left(N_{a}-I_{a}^{t}\right) a \int d a^{\prime} \frac{I_{a^{\prime}}^{t}}{N}+\alpha m\left(N_{a}-I_{a}^{t}\right) \int d a^{\prime} \frac{I_{a^{\prime}}^{t} a^{\prime}}{N}
$$

where $N_{a}$ is the total number of individuals with activity rate $a$ (which is constant over time). Each term in the Eq. (6) has a clear physical interpretation. In fact, the number of infected nodes in the class $a$ at time $t+1$ is given by: the number of infected nodes in this class at time $t$ (first term), minus the number of nodes that recover and going back to the class $S_{a}$ (second term), plus the number of infected individuals generated when nodes in the class $S_{a}^{t}=N_{a}-I_{a}^{t}$ are active and connect with infected nodes in the other activity classes (third term), plus the number of infected nodes generated when nodes in the class $S_{a}^{t}$ are linked by active infected nodes in other activity classes.

Summing on all of the classes and ignoring the second order terms we can write:

$$
\int d a I_{a}^{t+1}=I^{t+1}=I^{t}-\mu I^{t}+\alpha m\langle a\rangle I^{t}+\alpha m \theta^{t}
$$

where $\theta^{t}=\int d a^{\prime} I_{a^{\prime}}^{t} a^{\prime}$. Multiplying both sides of Eq. (6) by $a$ and integrating we obtain:

$$
\theta^{t+1}=\theta^{t}-\mu \theta^{t}+\alpha m\left\langle a^{2}\right\rangle I^{t}+\alpha m\langle a\rangle \theta^{t} .
$$

In the continuous time limit, we can write Eqs. (6) and (8) in a differential form:

$$
\begin{aligned}
\partial_{t} I & =-\mu I+\alpha m\langle a\rangle I+\alpha m \theta, \\
\partial_{t} \theta & =-\mu \theta+\alpha m\left\langle a^{2}\right\rangle I+\alpha m\langle a\rangle \theta .
\end{aligned}
$$

The Jacobian matrix of this set of linear differential equations takes the form

$$
J=\left(\begin{array}{cc}
-\mu+\alpha m\langle a\rangle & \alpha \\
\alpha m\left\langle a^{2}\right\rangle & -\mu+\alpha m\langle a\rangle
\end{array}\right)
$$

and has eigenvalues

$$
\Lambda_{(1,2)}=\langle a\rangle \alpha m-\mu \pm \alpha m \sqrt{\left\langle a^{2}\right\rangle} .
$$

The threshold is obtained by requiring the largest eigenvalues to be larger than 0 , which leads to: 


$$
\frac{\alpha}{\mu} \geq \frac{1}{m} \frac{1}{\langle a\rangle+\sqrt{\left\langle a^{2}\right\rangle}} .
$$

Considering the per capita spreading rate $\beta=\alpha\langle k\rangle$ we can write the threshold for the $S I S$ process, $\xi^{S I S}$, as:

$$
\frac{\beta}{\mu} \geq \xi^{S I S} \equiv \frac{2\langle a\rangle}{\langle a\rangle+\sqrt{\left\langle a^{2}\right\rangle}} .
$$

In words, the epidemic threshold is function of the first and second moment of the activity distribution. Due to the co-evolution of the network structure and the spreading processes the threshold is not dependent on time-aggregated metrics such as the degree. It is defined by the interplay between the time scale of the contagion process and the convolution of the network time scales encoded in the moments of the activity distribution.

\subsection{Controlling contagion processes in activity driven networks}

We can now study different immunization strategies. Following Ref. [83] and what presented above for annealed and static networks we will consider three main strategies: random, global and local. In all the cases, we introduce a fraction $p$ of nodes as immunized. To account for this new class of nodes, we introduce a new compartment, $R$, in the classic SIS scheme. Thus, the Eq. 6 becomes:

$$
\begin{aligned}
I_{a}^{t+1} & =I_{a}^{t}-\mu I_{a}^{t}+\alpha m\left(N_{a}-I_{a}^{t}-R_{a}^{t}\right) a \int d a^{\prime} \frac{I_{a^{\prime}}^{t}}{N}+ \\
& +\alpha m\left(N_{a}-I_{a}^{t}-R_{a}^{t}\right) \int d a^{\prime} \frac{I_{a^{\prime}}^{t} a^{\prime}}{N}
\end{aligned}
$$

First, let us consider the random strategy (RS) in which a fraction $p$ of nodes is immunized with an uniform probability [83]. In this case, the system of equations describing the dynamic process in activity-driven networks can be obtained by setting $R_{a}=p N_{a}$. The epidemic threshold condition changes as

$$
\frac{\beta}{\mu} \geq \xi^{R S} \equiv \frac{1}{1-p} \frac{2\langle a\rangle}{\langle a\rangle+\sqrt{\left\langle a^{2}\right\rangle}}=\frac{\xi^{S I S}}{1-p} .
$$

Consistently, with what we found for annealed and static networks, when a fraction $p$ of nodes is randomly immunized, the epidemic threshold can be written as the threshold with no intervention, $\xi^{S I S}$, rescaled by the number of nodes still available to the spreading process. Indeed, immunizing random nodes is equivalent to rescale the per capita spreading rate by the fraction of available nodes $\beta \rightarrow \beta(1-p)$. Another important quantity is the critical value of immunized nodes, $p_{c}$, necessary to halt the contagion process. This quantity is a function of network's structure and the 
specific features of the contagion process. The explicit value of $p_{c}$ can be obtained by inverting Eq. 15. In Fig. 1-A, we show $p_{c}$ as a function of $\beta / \mu$. The values on the heat map are the average asymptotic density of infected nodes, $I_{\infty}^{p}$. The phase space of the diffusion process is divided into two different regions separated by the blue solid line that represents $p_{c}$ as derived by Eq. 15. In the region below the curve, the spreading process will take over, $p<p_{c}$. However, in the region above the curve, the fraction of removed/immunized nodes is enough to completely stop the diffusion process, $p \geq p_{c}$. To assess the efficiency of the immunization strategy in Fig. 1-D (green triangles) we plot, as a function of the density of removed nodes $p$, the ratio $I_{\infty}^{p} / I_{\infty}^{0}$ where $I_{\infty}^{0}$ is the asymptotic density of infected nodes when no-intervention is implemented. As shown clearly in the figure, the random strategy allows a reduction in the fraction of infected nodes just for large values of $p$.
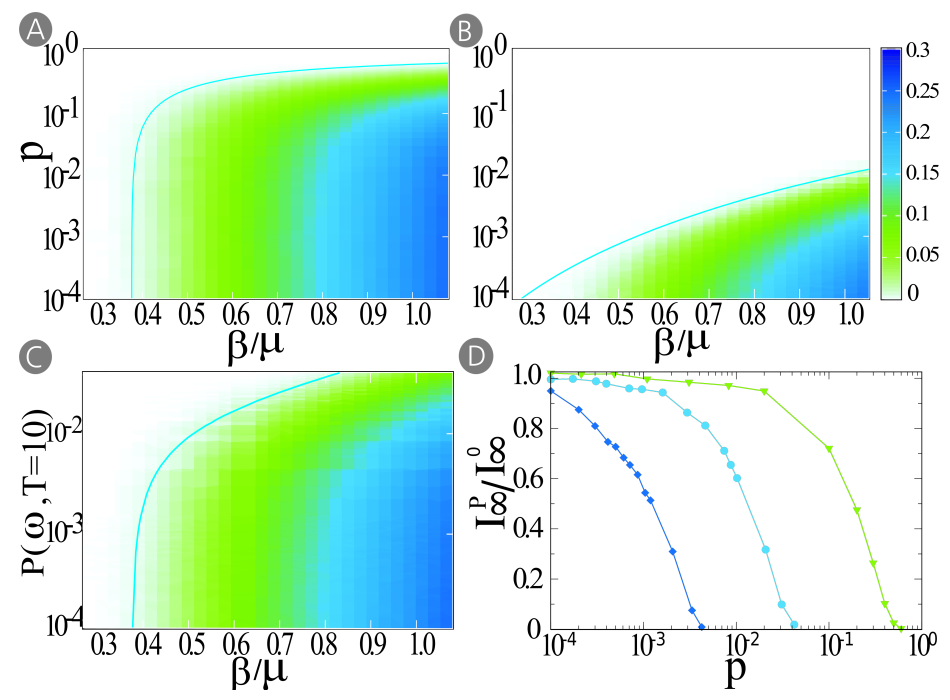

Fig. 1 Panels A, B, and C show the phase space of an SIS process under random, targeted, and egocentric control strategy, respectively. Considering $N=10^{4}, m=3, \varepsilon=10^{-3}$, activity distributed as $F(a) \sim a^{-2.2}$, we plot $I_{\infty}$ as a function of $\beta / \mu$ and $p$. Blue curves represent the critical value $p_{c}$. Panel D shows the comparison of the stationary state of a SIS model with and without control strategy, $I_{\infty}^{p} / I_{\infty}^{0}$, as a function of $p$ when $\beta / \mu=0.81$. In green triangles, we consider the random strategy, in dark blue diamonds the targeted strategy, and in blue circles the egocentric strategy. Each plot is made averaging $10^{2}$ independent simulations started with $1 \%$ of random seeds.

In activity-driven networks a natural way to implement a global immunization strategy is to target high activity nodes. Indeed, from the study of the epidemic threshold without intervention, we saw the importance of the moments of the activity distribution. By targeting high activity nodes we can reduce the average and the fluctuations of activity and thus move the threshold to higher values. Following this strategy we rank nodes in decreasing order of activity, immunizing the top ranking 
$p N$ nodes. This method is equivalent to fix a value $a_{c}$ so that any node with activity $a \geq a_{c}$ is immune to the contagion process ${ }^{1}$. Also, for this scheme, it is possible to derive the analytic expression for the epidemic threshold [83]:

$$
\frac{\beta}{\mu} \geq \xi^{T S} \equiv \frac{2\langle a\rangle}{\langle a\rangle^{c}+\sqrt{(1-p)\left\langle a^{2}\right\rangle^{c}}}
$$

where $\xi^{T S}$ indicates the threshold in the case of the targeted control strategy. In the above expression, we define $\left\langle a^{n}\right\rangle^{c}=\int_{\mathcal{E}}^{a_{c}} a^{n} F(a) d a$ as the moments of the activity distribution discounting the immunized nodes. Consistently to what found for annealed and static networks, Eq. (16) is not a simple rescaling of the original threshold expression and implies a drastic change in the behavior of the contagion process. In order to define the critical value of $p$ necessary to completely stop the spreading, we have to invert Eq. 16. The moments of the distribution of the remaining nodes are a function of $p$ through $a_{c}$ thus it is not possible to derive explicitly $p_{c}$. However, it can be easily evaluated numerically by solving the equation $\xi^{T S}-\beta / \mu=0$ for different values of $\beta / \mu$. In Fig. 1-B, we show $p_{c}$ (blue line) as a function of $\beta / \mu$. The efficiency of the targeted strategy is clear, which is also confirmed in Fig. 1-D (dark blue diamonds) where we plot the ratio $I_{\infty}^{p} / I_{\infty}^{0}$. Immunizing a very small fraction of the most active nodes is enough to stop the contagion process. Indeed, by immunizing just the top $1 \%$ of nodes is enough to halt the disease.

As discussed above, the network-wide knowledge required to implement targeted control strategies is generally not available [11]. In the case of evolving networks, this issue is even more pronounced as node's characterization depends on how long it is possible to observe the network dynamics. It is possible to generalized the acquaintance immunization strategy to activity-driven networks. A fraction $f$ of randomly selected nodes act as "probes". During an observation time $T$, we monitor their egonet generated by their interactions in the network. After the observation window, we select randomly a node in the egonet of each probe and immunizing it. For the sake of comparison with the previous control strategies, we define the fraction of actual immunized nodes as $p$ (in general $f \neq p^{2}$ ), and the epidemic starts after nodes have been immunized. In this scheme, the probability of immunization for one node with activity $a$ after a time step is:

$$
P_{a}=a f \int d a^{\prime} \frac{m N_{a^{\prime}}}{N}+f \int d a^{\prime} a^{\prime} \frac{m N_{a^{\prime}}}{N} \frac{1}{m} .
$$

The first term on the r.h.s. considers the probability that a node of class $a$ is active and reaches one of the probes; the second term, instead, takes into account the probability that one node of class $a$ gets a connection from one active probe. Solving the

\footnotetext{
${ }^{1}$ The value of $p$ and $a_{c}$ are linked by the relation $p=\int_{a_{c}}^{1} F(a) d a$

${ }^{2}$ In order to guarantee that a fraction $f$ of nodes is immunized the systems need to be observed for more than one time step. We define $T^{*}$ as the average time needed for all the probes to have at least one interaction with other nodes. For any observation time $T<T^{*}$ the fraction of immunized nodes will be in general $p \leq f$.
} 
integrals in Eq. (17), we can write $P_{a}=f(a m+\langle a\rangle)$. Thus, the probability of immunization of one node in the activity class $a$ after $t$ time steps is $P_{a}^{t}=1-\left(1-P_{a}\right)^{t}$, and therefore, summing over all the activity classes, we can estimate the total number of immunized individuals as $R^{T}=\sum_{a} N_{a} P_{a}^{T}=\sum_{a} N_{a}\left[1-\left(1-P_{a}\right)^{T}\right]$. The equation for $P_{a}$ does not consider the depletion of nodes in each class due to the immunization process. The formulation is then a good approximation for small $f$ and $T$, when the probability that a probe is selected more than once is very small. Replacing the expression for the removed/immunized individuals in the basic SIS equations yields the following epidemic threshold for the egocentric sampling strategy (ESS) [83]:

$$
\frac{\beta}{\mu} \geq \xi^{E S S} \equiv \frac{2\langle a\rangle}{\Psi_{1}^{T}+\sqrt{\Psi_{0}^{T} \Psi_{2}^{T}}},
$$

where we define $\Psi_{n}^{T}=\int d a a^{n}\left(1-P_{a}\right)^{T} F(a)$. This last integral is a function of the observing time window $T$, the probability of immunization of each class and the activity distribution. We evaluate each $\Psi$ term through numerical integration. As done for the other two cases, we define the critical value of $p$ by solving numerically the equation $\xi^{E S S}-\beta / \mu=0$ for different values of $\beta / \mu$. In Fig. 1-C, we show $p_{c}$ (blue solid line) as a function of $\beta / \mu$, and in Fig. 1-D we plot the ratio $I_{\infty}^{p} / I_{\infty}^{0}$ (blue dots). From these figures it is clear how this strategy is much more efficient than the random one, although not as performant as the targeted scheme. The efficiency of this strategy is due to the ability to reach active nodes by a local exploration done observing the systems for few time steps.

\subsection{Controlling contagion processes in real temporal networks}

Real world time-varying networks are characterized by a range of complex features such as heterogeneous activity patterns, correlations among nodes, persistence of links, and burstiness just to cite a few. For detailed reviews on the topic we refer the reader to Refs. [41, 42, 43]. Over the last years an increasing body of literature has been focused on the study of such properties and their relation to effective controlling strategies. Here, we will summarize some of the main results.

In their work, Prakash et al [77] used their analytical derivation of the epidemic threshold in a general time-varying network mentioned above, to study the efficiency of different controlling strategies in a real network obtained from the MIT Reality Mining project [84]. This dataset describes the interactions of 104 students recorded via Bluetooth. The authors consider several global and local strategies immunizing: 1) the top ranked nodes for degree in each temporal snapshot of the network, 2) the top ranked nodes for degree in the average adjacency matrix defined as $\left.A_{\text {average }}=T^{-1} \sum_{i=1, T} \mathbf{A}_{\mathbf{i}}, 3\right)$ nodes selected via the acquaintance method applied to the average adjacency matrix, 4 ) the nodes that sequentially (greedy method) induce the largest drop to the largest eigenvalue of the system matrix, 5) nodes in a 
sequence that provide the largest (optimal method) reduction to the largest eigenvalue of the system matrix. Interestingly, they found strategies 1, 2 and 4 to have similar performance respect the optimal strategy. Instead, the acquaintance strategy was found, not surprisingly, to perform significantly worst. It is important to notice that they used as objective metric to benchmark each strategy the change in the largest eigenvalue of the system matrix.

Lee et al [85] compared the acquaintance strategy against two variations of it, they proposed, which include the temporal dynamics on the networks. In particular, they considered strategies in which either the most recent or the most frequent contacts of randomly selected nodes are immunized. They studied the efficiency of each method in four different datasets: i) an online forum describing sexual interactions with prostitutes [86], ii) proximity interactions in hospital wards [87], email exchanges [88], and online dating [89]. They considered scenarios in which an immunization campaign can be applied in an initial time window of size $\Delta T$. Interestingly, they found that the two extensions of the acquaintance method have a larger impact on the disease spreading. Furthermore, in three of the four datasets the strategy based on the most recent contacts outperforms the method based on the most frequent contacts. As objective measure of performance they used $\Omega$ defined as "the average upper bound of outbreak size" obtained running epidemic spreading with $\alpha=1$ i.e. where the probability of infection per contact is one.

Starnini et al [90] studied several immunization strategies in different face-toface networks obtained via RFID tags in the SocioPatterns collaboration [91]. As done by Lee et al [85] they introduced an immunization campaign in the first $\Delta T$ time steps. They considered strategies in which the nodes to be immunized are selected considering: 1) the degree, 2) the betweenness, 3) the acquaintance method, 4) the frequency of activation of each $\operatorname{link}^{3}, 5$ ) the latest activated contacts in the time window ${ }^{3}$, and 6) randomly selected nodes. It is important to notice how this study contrasts global and local strategies considering both structural and temporal features. In the comparisons, the authors considered as objective metrics the speed of the spreading and the final number of infected nodes. Overall they found strategies that target nodes according to their degree and betweenness centrality to be more efficient. Furthermore, the authors observed a saturation effect for increasing sizes of the time window $\Delta t$. In principle, longer time windows should allow to gather more information and thus should be linked to better immunization performances. However, these observations show how this is true up to a certain point. The authors linked such unexpected behaviour to the emergence of central nodes at early stages in the system's dynamics. It is important to notice that even the considered datasets provide a very high temporal resolution, 20 seconds, they were recorded over typically a few days, thus they do not provide observations of long temporal trends of contact networks.

Tang et al [92] studied the spreading of malwares via bluetooth and efficient way to contain them. They considered three different datasets: the MIT reality mining data mentioned before, the interaction between researchers at the University of

\footnotetext{
${ }^{3}$ As proposed by Lee et al [85]
} 
Cambridge [93], and those between participants of a conference [94]. Interestingly, they found that strategies based on temporal betweenness centrality do not perform as expected. Although this metric provides the quantitative measure of the number of diffusion routes between nodes, the immunization of top ranked nodes does not necessarily stop the spreading as many alternative routes might exist. They proposed an immunization strategy in which the patch necessary to protect phones spread in the network is in competition with the malware. In their approach, the selection of nodes to immunize is done via the temporal closeness centrality measure which ranks node considering the speed at which each node can disseminate a message in the network. They showed how such strategy is consistently more efficient. Efficiency is measured in terms of the consumption of cellular network resources and time necessary to protect the system.

Liu et al [83] studied the efficiency of the three different strategies introduced in sub-section 3.2 in a mobile phone call data network. Interestingly, the numerical simulations confirm qualitatively the analytical results obtained in activity driven networks. In particular, the strategy that target high active nodes is the most efficient, followed by the generalization of the acquaintance methods: the egocentric sampling strategy. The objective measure adopted to judge the efficiency of each strategy is the ratio between the stationary state of a SIS model with and without control strategy, i.e. $I_{\infty}^{p} / I_{\infty}^{0}$.

\section{Conclusions and outlook}

We presented a summary of strategies to control contagion processes in annealed, static, and time-varying networks. In doing so, we considered a range of different analytical and numerical results. We observed how heterogeneities that characterize many features of real world networks make targeted global strategies extremely efficient. However, these strategies assume complete knowledge of the networks' topology and their dynamical evolution. Local strategies based on local exploration and sample of the networks are in general less efficient that global methods but are more realistic and provide a higher performance respect to random strategies.

We considered scenarios where the dynamics of networks (temporal evolution of networks' structure) affect the dynamics on networks (contagion processes). In general also the opposite is true. For example, the spreading of an infectious disease might induce changes in the evolution of a contact network $[95,96]$ or the spreading of information on an online social network might affect its structure and evolution [97]. In this case the dynamics on the networks affect the dynamics of the network. In the literature the study of this reverse problem (respect to what we considered here) is found under the umbrella of adaptive networks. We invite the interested reader to several other chapters of this book [ADD REFERENCES] for details.

Generally speaking, the dynamics of and on networks are interwinded. Networks are subject to natural temporal dynamics which are perturbed by contagion pro- 
cesses. In turn, contagion processes are subject to the natural evolution of networks and are affected by the changes they induce. The understanding of these intricacies is still very limited and requires the development of methods to model and mathematically describe complex coupled dynamics, possibly acting at different timescales. Furthermore, it requires the availability of high resolution data that capture all these processes and their interplay. Despite the unprecedented access to large-scale datasets of human interactions in recent times, the observation of the feedback loop between the dynamics of and on networks is typically indirect and thus the casual link between the two, necessary to inform or test model, is often missing.

We believe that addressing all these challenges is of extreme importance to better understand the spreading of contagion processes on real networks and to develop efficient methods to control them. Doing so will require an interdisciplinary effort from a set of disciplines ranging from network and data science to sociology and applied mathematics.

Acknowledgements The results presented in section 3.2 are adapted from Ref. [83] and obtained in collaboration with S. Liu and A. Vespignani.

\section{References}

1. A. Barrat, M. Barthélemy, and A. Vespignani. Dynamical Processes on Complex Networks. Cambridge Univesity Press, 2008.

2. M.E.J. Newman. Networks. An Introduction. Oxford Univesity Press, 2010.

3. R. Cohen and S. Havlin. Complex Networks: Structure, Robustness and Function. Cambridge University Press, Cambridge, 2010.

4. C.T. Butts. Revisting the foundations of network analysis. Science, 325:414-416, 2009.

5. Bruno Gonçalves and Nicola Perra. Social phenomena: From data analysis to models. Springer, 2015.

6. A. Vespignani. Predicting the behavior of techno-social systems. Science, 325:425-428, 2009.

7. S Fortunato. Community detection in graphs. Physics Reports, 486:75-174, 2010.

8. Zhen Wang, Chris T. Bauch, Samit Bhattacharyya, Alberto d?Onofrio, Piero Manfredi, Matja Perc, Nicola Perra, Marcel Salath, and Dawei Zhao. Statistical physics of vaccination. Physics Reports, pages -, 2016.

9. M. Kitsak, L. K. Gallos, S. Havlin, and H. A. Makse. Identification of infuential spreaders in complex networks. Nature Physics, 6:888, 2010.

10. R Pastor-Satorras and A Vespignani. Immunization of complex networks. Phys. Rev. E, 63:036104, 2002.

11. R. Cohen, S. Havlin, and D. ben Avraham. Efficient immunization strategies for computer networks and populations. Phys Rev. Lett., 91, 2003.

12. M. Morris. Telling tails explain the discrepancy in sexual partner reports. Nature, 365:437, 1993.

13. M. Morris. Sexually Transmitted Diseases, K.K. Holmes, et al. Eds. McGraw-Hill, 2007.

14. A. Clauset and N. Eagle. Persistence and periodicity in a dynamic proximity network. In DIMACS Workshop on Computational Methods for Dynamic Interaction Networks, pages 15, 2007.

15. A. Vespignani. Modeling dynamical processes in complex socio-technical systems. Nature Physics, 8:32-30, 2012. 
16. Luis E. C. Rocha, Fredrik Liljeros, and Petter Holme. Simulated epidemics in an empirical spatiotemporal network of 50,185 sexual contacts. PLoS Comput Biol, 7(3):e1001109, 03 2011.

17. Lorenzo Isella, Juliette Stehlé, Alain Barrat, Ciro Cattuto, Jean-François Pinton, and Wouter Van den Broeck. What's in a crowd? analysis of face-to-face behavioral networks. J. Theor. Biol, 271:166, 2011.

18. Juliette Stehlé, Nicolas Voirin, Alain Barrat, Ciro Cattuto, Vittoria Colizza, Lorenzo Isella, Corinne Régis, Jean-François Pinton, Nagham Khanafer, Wouter Van den Broeck, and Philippe Vanhems. Simulation of an seir infectious disease model on the dynamic contact network of conference attendees. BMC Medicine, 9(87), 2011.

19. M. Karsai, M. Kivelä, R. K. Pan, K. Kaski, J. Kertész, A.-L. Barabási, and J. Saramäki. Small but slow world: How network topology and burstiness slow down spreading. Phys. Rev. E, 83:025102, Feb 2011.

20. Giovanna Miritello, Esteban Moro, and Rubén Lara. Dynamical strength of social ties in information spreading. Phys. Rev. E, 83:045102, Apr 2011.

21. M. Kivela, R. Kumar Pan, K. Kaski, J. Kertesz, Jari Saramaki, and M. Karsai. Multiscale analysis of spreading in a large communication network. arXiv:1112.4312v1, 2011.

22. N. Fujiwara, J. Kurths, and A. Díaz-Guilera. Synchronization in networks of mobile oscillators. Physical Review E, 83(2):025101, 2011.

23. R. Parshani, M. Dickison, R. Cohen, H. E. Stanley, and S. Havlin. Dynamic networks and directed percolation. EPL (Europhysics Letters), 90(3):38004, 2010.

24. Paolo Bajardi, Alain Barrat, Fabrizio Natale, Lara Savini, and Vittoria Colizza. Dynamical patterns of cattle trade movements. PLoS ONE, 6(5):e19869, 052011.

25. A. Panisson, A. Barrat, C. Cattuto, W. Van den Broeck, G. Ruffo, and R. Schifanella. On the dynamics of human proximity for data diffusion in ad-hoc networks. Ad Hoc Networks, 10, 2011.

26. Andrea Baronchelli and Albert Díaz-Guilera. Consensus in networks of mobile communicating agents. Phys. Rev. E, 85:016113, Jan 2012.

27. Michele Starnini, Andrea Baronchelli, Alain Barrat, and Romualdo Pastor-Satorras. Random walks on temporal networks. Phys. Rev. E, 85:056115, May 2012.

28. R. Pfitzner, I. Scholtes, A. Garas, C.J Tessone, and F. Schweitzer. Betweenness preference: Quantifying correlations in the topological dynamics of temporal networks. Phys. Rev. Lett., 110:19, 2013.

29. M. Karsai, N. Perra, and A. Vespignani. Time varying networks and the weakness of strong ties. Scientific Reports, 4:4001, 2014.

30. T. Hoffmann, M.A. Porter, and R. Lambiotte. Generalized master equations for non-poisson dynamics on networks. Physical Review E, 86:046102, 2012.

31. Z. Toroczkai and H. Guclu. Proximity networks and epidemics. Physica A, 378.

32. N. Perra, B. Gonçalves, R. Pastor-Satorras, and A. Vespignani. Time scales and dynamical processes in activity driven networks. Scientific Reports, 2:469, 2012.

33. B Ribeiro, N. Perra, and A. Baronchelli. Quantifying the effect of temporal resolution on time-varying networks. Scientific Reports, 3:3006, 2013.

34. N. Perra, A. Baronchelli, D Mocanu, B. Gonçalves, R. Pastor-Satorras, and A. Vespignani. Random walks and search in time varying networks. Phys. Rev. Lett., 109:238701, 2012.

35. S. Liu, A. Baronchelli, and N. Perra. Contagion dynamics in time-varying metapopulations networks. Phy. Rev. E, 87(032805), 2013.

36. M. Starnini and R. Pastor-Satorras. Topological properties of a time-integrated activity-driven network. Phys. Rev. E, 87:062807, 2013.

37. T. Takaguchi, N. Sato, K. Yano, and N. Masuda. Importance of individual events in temporal networkss. New J. Phys., 14:093003, 2012.

38. Taro Takaguchi, Naoki Masuda, and Petter Holme. Bursty communication patterns facilitate spreading in a threshold-based epidemic dynamics. PloS one, 8(7):e68629, 2013.

39. Petter Holme and Fredrik Liljeros. Birth and death of links control disease spreading in empirical contact networks. Scientific reports, 4, 2014. 
40. Petter Holme and Naoki Masuda. The basic reproduction number as a predictor for epidemic outbreaks in temporal networks. PloS one, 10(3):e0120567, 2015.

41. P. Holme and J. Saramäki. Temporal networks. Phys. Rep., 519:97, 2012.

42. Petter Holme. Modern temporal network theory: a colloquium. The European Physical Journal B, 88(9):1-30, 2015.

43. Naoki Masuda and Renaud Lambiotte. A guide to temporal networks. World Scientific, 2016.

44. W. O. Kermack and A. G. McKendrick. A contribution to the mathematical theory of epidemics. Proc. R. Soc. A, 115:700, 1927.

45. M.J. Keeling and P. Rohani. Modeling Infectious Disease in Humans and Animals. Princeton University Press, 2008.

46. R. Pastor-Satorras and A. Vespignani. Epidemic spreading in scale-free networks. Phys. Rev, Lett., 86:3200, 2001.

47. Y. Wang, D. Chakrabarti, G. Wang, and C Faloutsos. Epidemic spreading in real networks: An eigenvalue viewpoint. In Proc 22nd International Symposium on Reliable Distributed Systems, pages 25-34, 2003.

48. C Castellano and R Pastor-Satorras. Thresholds for epidemic spreading in networks. Phys. Rev. Lett., 105:218701, 2010.

49. R. Durrett. Some features of the spread of epidemics and information on a random graph. Proc. Nat. Acad. Sci., 107:4491-4498, 2010.

50. Alexander V Goltsev, Sergey N Dorogovtsev, JG Oliveira, and Jose FF Mendes. Localization and spreading of diseases in complex networks. Physical review letters, 109(12):128702, 2012.

51. M. Boguñá, C. Castellano, and R. Pastor-Satorras. Nature of the epidemic threshold for the susceptible-infected-susceptible dynamics in networks. Physical review letters, 111(6):068701, 2013.

52. C Castellano and R Pastor-Satorras. Thresholds for epidemic spreading in networks. Physical review letters, 105(21):218701, 2010.

53. H.K. Lee, P.S. Shim, and J.D. Noh. Epidemic threshold of the susceptible-infected-susceptible model on complex networks. Physical Review E, 87(6):062812, 2013.

54. R. Albert, H. Jeong, and A.L. Barabási. Error and attack tolerance of complex networks. Nature, 406:378, 2000.

55. Romualdo Pastor-Satorras and Alessandro Vespignani. Epidemics and immunization in scalefree networks, pages 111-130. Wiley-VCH Verlag GmbH Co. KGaA, 2005.

56. Nicola Perra and Santo Fortunato. Spectral centrality measures in complex networks. Physical Review E, 78(3):036107, 2008.

57. Laurent Hébert-Dufresne, Antoine Allard, Jean-Gabriel Young, and Louis J. Dubé. Global efficiency of local immunization on complex networks. Scientific Reports, 3, July 2013.

58. Gnana Thedchanamoorthy, Mahendra Piraveenan, Shahadat Uddin, and Upul Senanayake. Influence of vaccination strategies and topology on the herd immunity of complex networks. Social Network Analysis and Mining, 4(1):213, 2014.

59. Yiping Chen, Gerald Paul, Shlomo Havlin, Fredrik Liljeros, and H. Eugene Stanley. Finding a better immunization strategy. Phys. Rev. Lett., 101:058701, Jul 2008.

60. Attack vulnerability of complex networks. Phys. Rev. E, 65(5):056109+, May 2002.

61. C. M. Schneider, T. Mihaljev, and H. J. Herrmann. Inverse targeting ?An effective immunization strategy. EPL (Europhysics Letters), 98(4):46002+, May 2012.

62. Scott L. Feld. Why Your Friends Have More Friends Than You Do. American Journal of Sociology, 96(6):1464-1477, 1991.

63. Nathan O. Hodas, Farshad Kooti, and Kristina Lerman. Friendship Paradox Redux: Your Friends Are More Interesting Than You. In Proceedings of 7th International Conference on Weblogs and Social Media, April 2013.

64. Silvio Lattanzi and Yaron Singer. The Power of Random Neighbors in Social Networks. In Proceedings of the Eighth ACM International Conference on Web Search and Data Mining, WSDM '15, pages 77-86, New York, NY, USA, 2015. ACM.

65. N. Madar, T. Kalisky, R. Cohen, D. ben Avraham, and S. Havlin. Immunization and epidemic dynamics in complex networks. The European Physical Journal B, 38(2):269-276, 2004. 
66. Zoltán Dezsô and Albert-László Barabási. Halting viruses in scale-free networks. Phys. Rev. E, 65:055103, May 2002.

67. L K Gallos, F Liljeros, P Argyrakis, A Bunde, and S Havlin. Improving immunization strategies. Phys. Rev. E, 75:045104(R), 2007.

68. Gouhei Tanaka, Chiyori Urabe, and Kazuyuki Aihara. Random and targeted interventions for epidemic control in metapopulation models. Scientific Reports, 4:5522 EP -, 072014.

69. Kai Gong, Ming Tang, Pak M. Hui, Hai F. Zhang, Do Younghae, and Ying-Cheng Lai. An Efficient Immunization Strategy for Community Networks. PLOS ONE, 8(12):1-11, December 2013.

70. M. Salathé and J.H. Jones. Dynamics and control of diseases in networks with community structure. PLoS Comput Biol, 6(4):e1000736, 2010.

71. K.T.D. Eames. Modelling disease spread through random and regular contacts in clustered populations. Theoretical population biology, 73(1):104-111, 2008.

72. T. Smieszek, L. Fiebig, and R.W. Scholz. Models of epidemics: when contact repetition and clustering should be included. Theoretical Biology and Medical Modelling, 6(1):1, 2009.

73. J.C. Miller. Spread of infectious disease through clustered populations. Journal of the Royal Society Interface, pages rsif-2008, 2009.

74. T. Britton, M. Deijfen, A.N. Lagerås, M. Lindholm, et al. Epidemics on random graphs with tunable clustering. Journal of Applied Probability, 45(3):743-756, 2008.

75. Alessio Cardillo, Catalina Reyes-Suárez, Fernando Naranjo, and Jesús Gómez-Gardeñes. Evolutionary vaccination dilemma in complex networks. Phys. Rev. E, 88:032803, Sep 2013.

76. Ellsworth Campbell and Marcel Salathé. Complex social contagion makes networks more vulnerable to disease outbreaks. Scientific Reports, 3:1905 EP -, 052013.

77. B.A. Prakash, H. Tong, N. Valler, M. Faloutsos, and C. Faloutsos. Virus propagation on timevarying networks: Theory and immunization algorithms. In Joint European Conference on Machine Learning and Knowledge Discovery in Databases, pages 99-114. Springer, 2010

78. E. Valdano, L. Ferreri, C. Poletto, and V. Colizza. Analytical computation of the epidemic threshold on temporal networks. Physical Review X, 5(2):021005, 2015.

79. M.V. Tomasello, N. Perra, C.J. Tessone, M. Karsai, and F. Schweitzer. The role of endogenous and exogenous mechanisms in the formation of r\&d networks. Scientific reports, 4, 2014.

80. E. Ubaldi, N. Perra, M. Karsai, A. Vezzani, R. Burioni, and A. Vespignani. Asymptotic theory of time-varying social networks with heterogeneous activity and tie allocation. Scientific Reports, 6, 2016.

81. E. Ubaldi, A. Vezzani, M. Karsai, N. Perra, and R. Burioni. Burstiness and tie reinforcement in time varying social networks. arXiv preprint arXiv:1607.08910, 2016.

82. M. Starnini and R. Pastor-Satorras. Topological properties of a time-integrated activity-driven network. Physical Review E, 87(6):062807, 2013.

83. S. Liu, N. Perra, M. Karsai, and A. Vespignani. Controlling contagion processes in activity driven networks. Physical review letters, 112(11):118702, 2014.

84. A Pentland, N Eagle, and D Lazer. Inferring social network structure using mobile phone data. Proceedings of the National Academy of Sciences (PNAS), 106(36):15274-15278, 2009.

85. S. Lee, L.E.C. Rocha, F. Liljeros, and P. Holme. Exploiting temporal network structures of human interaction to effectively immunize populations. PLoS ONE, 7:e36439, 2012.

86. LEC Rocha, F Liljeros, and P Holme. Information dynamics shape the sexual networks of internet-mediated prostitution. Proceedings of the National Academy of Sciences, 107(13):5706-5711, 2010.

87. F Liljeros, J Giesecke, and P Holme. The contact network of inpatients in a regional healthcare system. a longitudinal case study. Mathematical Population Studies, 14(4):269-284, 2007.

88. Jean-Pierre Eckmann, Elisha Moses, and Danilo Sergi. Entropy of dialogues creates coherent structures in e-mail traffic. Proceedings of the National Academy of Sciences of the United States of America, 101(40):14333-14337, 2004.

89. P Holme, CR Edling, and F Liljeros. Structure and time evolution of an internet dating community. Social Networks, 26(2):155-174, 2004. 
90. M. Starnini, A. Machens, C. Cattuto, A. Barrat, and R. Pastor-Satorras. Immunization strategies for epidemic processes in time-varying contact networks. Journal of Theoretical Biology, 337:89-100, 2013.

91. Sociopatterns. http://www.sociopatterns.org/.

92. J. Tang, C. Mascolo, M. Musolesi, and V. Latora. Exploiting temporal complex network metrics in mobile malware containment. In World of Wireless, Mobile and Multimedia Networks (WoWMoM), 2011 IEEE International Symposium on a, pages 1-9. IEEE, 2011.

93. Kiran K Rachuri, Mirco Musolesi, Cecilia Mascolo, Peter J Rentfrow, Chris Longworth, and Andrius Aucinas. Emotionsense: a mobile phones based adaptive platform for experimental social psychology research. In Proceedings of the 12th ACM international conference on Ubiquitous computing, pages 281-290. ACM, 2010.

94. James Scott, Richard Gass, Jon Crowcroft, Pan Hui, Christophe Diot, and Augustin Chaintreau. CRAWDAD dataset cambridge/haggle (v. 2009-05-29). Downloaded from http://crawdad.org/cambridge/haggle/20090529, May 2009.

95. S. Funk, M. Salathé, and V.A.A. Jansen. Modelling the influence of human behaviour on the spread of infectious diseases: a review. Journal of the Royal Society Interface, 7(50):12471256, 2010.

96. Z. Wang, M.A. Andrews, Z.X. Wu, L. Wang, and C.T. Bauch. Coupled disease-behavior dynamics on complex networks: A review. Physics of life reviews, 15:1-29, 2015.

97. L. Weng, J. Ratkiewicz, N. Perra, B. Gonçalves, C. Castillo, F. Bonchi, R. Schifanella, F. Menczer, and A. Flammini. The role of information diffusion in the evolution of social networks. In Proceedings of the 19th ACM SIGKDD international conference on Knowledge discovery and data mining, pages 356-364. ACM, 2013. 